\title{
Activation Profile of Pectoralis Major, Deltoid and Supraspinatus Muscle in Brachial Plexus Injury Patient with Shoulder Subluxation Using Shoulder Sling - A Case Report
}

\author{
Poly Ghosh*, Lenka PK and Abhishek Biswas \\ Department of National Institute for Locomotor Disabilities (Divyangjan), India \\ *Corresponding author: Poly Ghosh, Department of National Institute for Locomotor Disabilities (Divyangjan), India
}

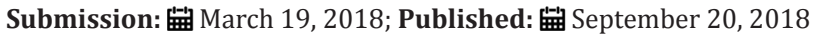

\section{Introduction}

Brachial plexus lesions frequently lead to significant physical disability, psychologic distress, and socioeconomic hardship. Adult brachial plexus injuries can be caused by various mechanisms, including penetrating injuries, falls, and motor vehicle trauma. Often the diagnosis is delayed or ignored as the practitioner waits for some recovery. Expedient diagnosis and testing is the best means of maximizing functional return. Evaluators must remember that muscles will begin to undergo atrophy and lose motor end plates as soon as the proximal injury occurs [1]. The vast majority of brachial plexus injuries involve denervation of the shoulder's supporting musculature. A weak shoulder girdle allows subluxation of the glenohumeral joint. The shoulder joint is inherently unstable, although the capsule and ligaments play a supporting role in joint stability, the majority of support is provided through active contraction of the supraspinatus muscle. Therefore, significant weakening of the supraspinatus leads to shoulder subluxation [2]. However, electromyography (EMG) study is required for muscle weakness by nerve damage. The study of muscle function through EMG is an established method for quantifying muscle activity through electrical activity [3].

In brachial plexus injury as nerve damaged occurred so electromyography is the best option to assess the muscle function [4]. Inappropriate muscle activation has been observed to contribute to shoulder instability and studies have reported that muscles can have both a stabilizing and destabilizing role [3]. Shoulder orthoses can be used to counteract the shoulder subluxation [2]. In BPI, shoulder subluxation is caused by supraspinatus muscle but whether any other muscle is involved to assess that EMG study is required. Studies suggested that in immobilized shoulder, some amount of EMG activity is present. In shoulder orthosis all of the shoulder muscles are immobilized. So, aim of this case report is to find out the patterns of shoulder muscle activity (deltoid, supraspinatus and pectoralis minor) in shoulder subluxation patient before and after using shoulder sling.

\section{Methodology}

\section{Case report}

A 40 years male brachial plexus injured patient reported to Department of Prosthetics and orthotics, National institute for Locomotor Disabilities, Kolkata, with chief complaint of shoulder sublaxation and unable to use his left upper limb for activities of daily living. On observation, patient was having shoulder sublaxation.

\section{History}

1 year before patients got injured as someone pull his left upper limb to help him to climb stairs and he felt sudden pain on shoulder joint. After sometimes he felt numbness in his left upper limb, he was immediately taken to local hospital and doctor diagnosed him as a brachial plexus injury, from then he was undergone treatment. Presently he came to our institute for further treatment Figure 1.

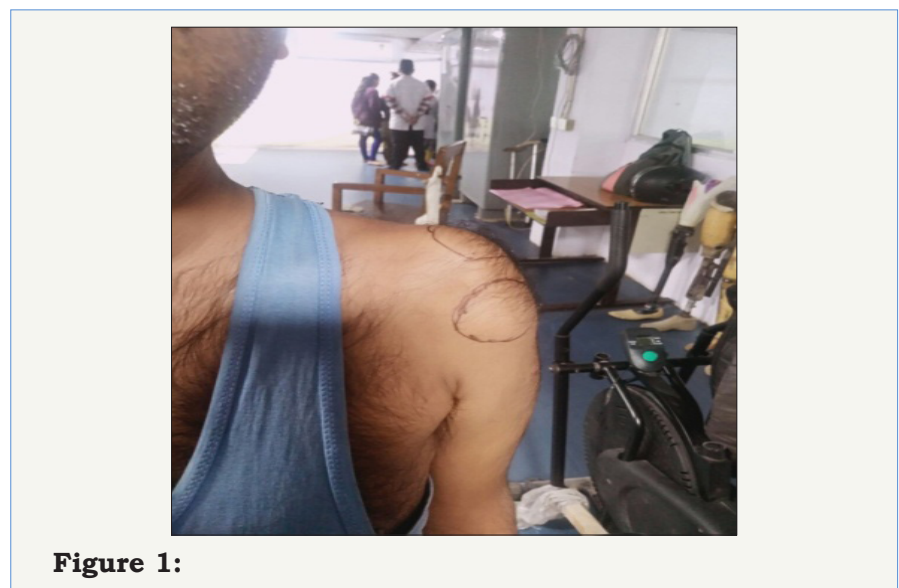


On examination:

A. Shoulder subluxation $=3 \mathrm{~cm}$

B. Antero-inferior shoulder subluxation

C. Elbow flexors power good to normal

D. Hand and wrist muscle (flexors) power are good to normal

E. Atrophy of deltoid and rotator cuff muscle

F. Loss of Shoulder abduction, flexion, rotation and extension

G. Active shoulder elevation possible.

H. Upper trunk and C5-C6 injury

After Assessment, patient was prescribed standard shoulder sling. After using of shoulder sling for 1 week, shoulder muscle activation profile using EMG was taken with and without shoulder sling.

\section{Methods of measuring shoulder girdle muscle activation}

Muscle activity was recorded by Ad instrument power lab EMG analyzer using Surface electrode (Agcl). Data were recorded for 2 minutes and data analyzed for middle 1 minute as first 30 seconds and last 30seconds counted as noise.

Data was taken in four condition:

A. Shoulder joint in resting condition (without sling support)

B. Actively elevated shoulder position (without sling support)

C. Shoulder joint in Resting condition (with sling support)

D. Actively elevated shoulder position (with sling support)

Protocol of data collection: During data collection, the Patients were asked to sit on a chair in a relaxed condition.

\section{SEMG (surface electromyography) pre-recording proce- dure}

Choice of electrode: Round shaped Silver/Silver Chloride (Ag/ $\mathrm{AgCl}$ ) surface electrode was used to record the EMG and attached with doubled adhesive tape to the patient. Conductive electrode gel was required to increase conductivity of the EMG signal Figure 2.

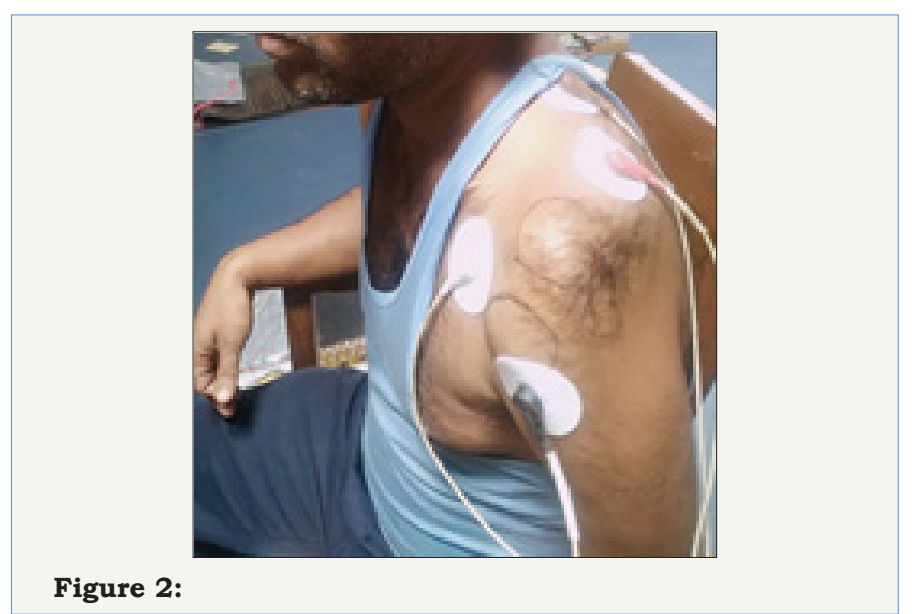

Preparation of skin: To lower the skin resistance, the skin preparation including washing, wiping was done to have better EMG recordings. After the preparation of skin, surface electrode was placed in the forearm muscle belly and parallel to the muscle fiber, so that the maximum signal can be collected. AgCl gel was then applied to the to reduce skin impedance.

Electrode placement: After scrubbing the area and properly cleaning the skin with alcohol, the surface electrode was placed lengthwise over the location of pectoralis minor, deltoid and supraspinatus. Electrodes location was determined using palpation while the subjects were alternatively activating and relaxing their muscles. Electrodes were placed on the thickest part of the muscle belly. Electrode must have good contact with the skin and must be aligned along the general direction of muscle fibers. The earth electrode was placed on the forearm.

One ground electrode

A. $\quad$ Channel 1-black placed over deltoid anterior fiber \& white placed pectoralis minor muscle

B. Channel 2-Red \& brown-placed over supraspinatus

\section{SEMG data recording procedure}

EMG signal data while simultaneously displaying up to 3 separate channels of EMG Signals to a standard computer screen for visual inspection (Figure 3). RMS amplitude was used to describe signal strength of SEMG signals.

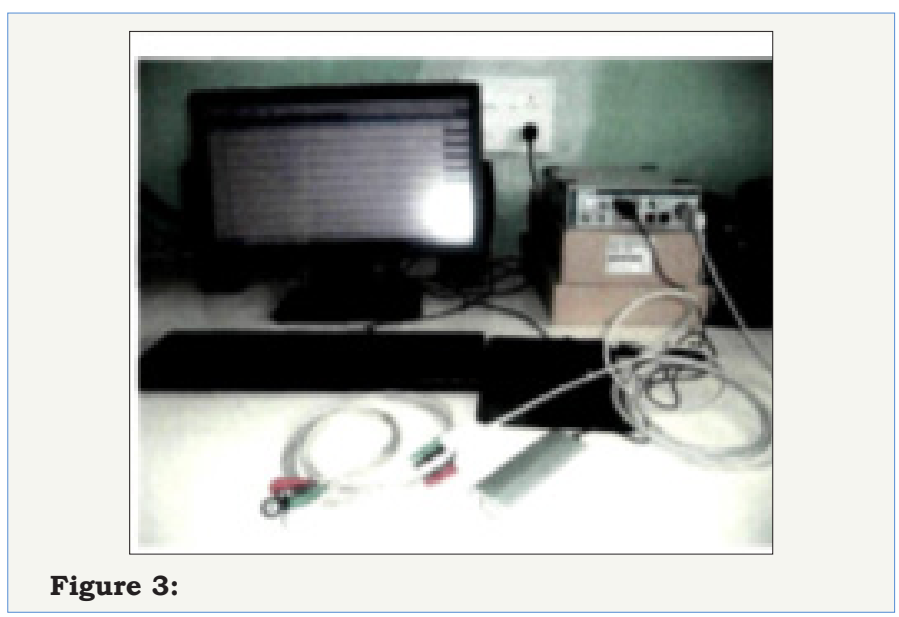

\section{Result and Discussion}

A sling or other method of support is needed for patients with injuries to the brachial plexus nerves That control the shoulder and arm muscles. Damage to these Nerves can result in Partial or total loss of motor function of the arm and sublaxation of the humeral head from the shoulder socket (Figure 4). Brachial plexus patients must rely on shoulder and arm supporting splints to fix this subluxation. Result showed in resting position without any sling support, Pectoralis and deltoid muscle $(0.8036 \mathrm{mV})$ are more active and stronger than supraspinatus $(0.064 \mathrm{mV})$, supraspinatus is also active but that is negligible (Table 1). During active elevation supraspinatus muscle $(0.3371 \mathrm{mV})$ is stronger than pectoralis and deltoid muscle $(0.303 \mathrm{mV})$ but very less in comparison. But with sling 
support, in resting condition all the muscles $(0.0637 \& 0.0889 \mathrm{mV})$ amplitude is less but in elevation with sling support all the muscles are equally active. RMS value has increased in spuraspinatus muscle with sling support in both condition which suggest that sling has effects on supraspinatus muscle. In this case patient was having brachial plexus injury, in spite of that muscles are showing some effects in EMG, that means muscles are not totally paralyzed but partially paralyzed and sling has some support effects (Table 2). From this study it may be concluded that, use of shoulder sling play a vital role in prevention of shoulder sublaxation and help in muscle activity augmentation.

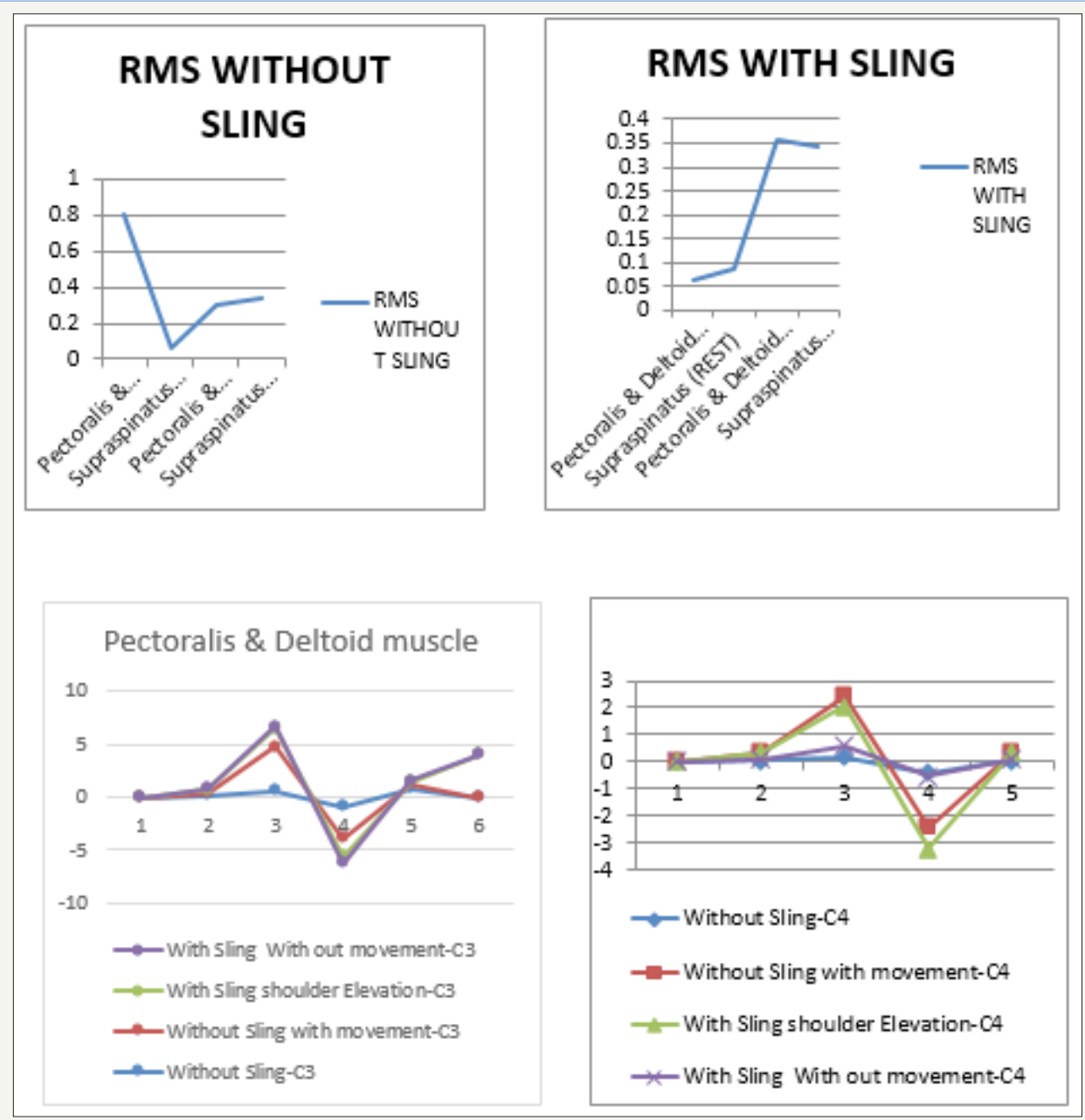

Figure 4:

Table1: Ch3=Pectoralis \& deltoid muscle group, Ch4=supraspinatus muscle, unit of measurement=mV.

\begin{tabular}{|c|c|c|c|c|}
\hline Parameters & Shoulder Joint in Resting Position (without sling) & \multicolumn{2}{|c|}{ Actively Elevated Shoulder Position (without sling) } \\
\hline & Ch3 & Ch4 & Ch3 & $0037 \pm .34$ \\
\hline Mean \pm SD & $-0247 \pm .08$ & $0206 \pm .06$ & $-0295 \pm .3$ & 2.4325 \\
\hline Max. Value & 0.6219 & 0.1675 & 4.1769 & -2.4413 \\
\hline Min. Value & -0.9081 & -0.4475 & -3.0144 & 0.3371 \\
\hline RMS & 0.8036 & 0.064 & 0.303 & \\
\hline
\end{tabular}

Table 2: Ch3=Pectoralis \& deltoid muscle group, Ch4=supraspinatus muscle, unit of measurement=mV.

\begin{tabular}{|c|c|c|c|c|}
\hline Parameters & Shoulder Joint in Resting Condition (with sling Support) & \multicolumn{2}{|c|}{ Actively Elevated Shoulder Position (with sling Support) } \\
\hline & Ch3 & Ch4 & Ch3 & Ch4 \\
\hline Mean \pm SD & $-03 \pm .05$ & $024 \pm .08$ & $-012 \pm .35$ & $021 \pm .34$ \\
\hline Max. Value & 0.2144 & 0.6075 & 1.6219 & 2.065 \\
\hline
\end{tabular}




\begin{tabular}{|c|c|c|c|c|}
\hline Min. Value & -0.6356 & -0.54 & -1.7656 & -3.21 \\
\hline RMS & 0.0637 & 0.0889 & 0.3587 & 0.3446 \\
\hline
\end{tabular}

\section{Acknowledgement}

The authors sincerely Director Dr. Abhishek Biswas of National Institute for Locomotor Disabilities (Divyangjan) for allowing to use Gait lab.

\section{References}

1. Moran SL, Steinmann SP, Shin AY (2005) Adult brachial plexus injuries: mechanism, patterns of injury, and physical diagnosis. Hand clinics 21(1): $13-24$
2. John D Hsu, John W Michael, John R Fisk (2008) AAOS atlas of orthoses and assistive device $\left(4^{\text {th }}\right.$ edn). American Academy of Orthopedic Surgeons, Elsevier Health Sciences, USA.

3. Jaggi A, Noorani A, Malone A, Cowan J, Lambert S, et al. (2012) Muscle activation patterns in patients with recurrent shoulder instability. International Journal of Shoulder Surgery 6(4): 101-107.

4. Raghig H, Young GB, Hammond R, Nicolle M (2010) A comparison of EMG and muscle biopsy in ICU weakness. Neurocritical care 13 (3): 326330. (c) (i) Creative Commons Attribution 4.0

For possible submissions Click Here
Submit Article

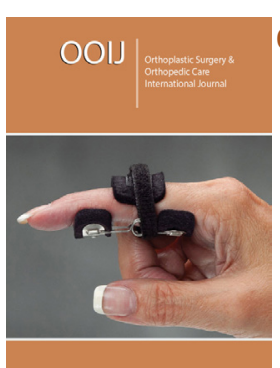
Orthoplastic Surgery \& Orthopedic Care International
Journal

\section{Benefits of Publishing with us}

- High-level peer review and editorial services

- Freely accessible online immediately upon publication

- Authors retain the copyright to their work

- Licensing it under a Creative Commons license

- Visibility through different online platforms 